\title{
Extensive Analysis of Clustered Routing Protocols For Heteregeneous Sensor Networks
}

\author{
Y. Yazid ${ }^{1}$, I. Ez-zazi ${ }^{1}$, M. Salhaoui ${ }^{1,2}$, M. Arioua ${ }^{1}$, A. El Oualkadi ${ }^{1}$, A. Guerrero González ${ }^{2}$ \\ \{yazidyassine8, mounir.arioua, eloualkadi \}@gmail.com \\ ${ }^{1}$ Laboratory of Information and Communication Technologies, National School of Applied Sciences, \\ Abdelmalek Essaadi University, Tangier, Morocco \\ ${ }^{2}$ Polytechnic University of Cartagena, Spain
}

\begin{abstract}
Energy efficiency has been widely considered as a critical issue in wireless sensor networks (WSN) due to the limited battery powered nodes. Therein, communication process is the most energy demanding in sensor nodes. Accordingly, using energy aware routing protocols to minimize the communication cost and prolong the network lifetime is of paramount importance. In this paper, an extensive analysis of routing protocols based on clustering approach is carried out. We have mainly focused on the performant clustered routing protocols dedicated for heterogeneous WSNs namely Stable Election Protocol (SEP), and its most recent based routing algorithms. The performances of these protocols based on various parameters such as energy efficiency, network lifetime, throughput and stability have been studied. The simulation results show that Threshold SEP clustered routing algorithm provides the best performance in terms of energy, network lifetime and throughput compared to other SEP routing algorithms.
\end{abstract}

Keywords: WSN, Energy Consumption, Routing, Clustering, SEP.

\section{Introduction}

In the last few years, wireless sensor networks (WSN) have experienced a tremendous upsurge. Notably, this technology has found application in a large number of fields, e.g. industry, monitoring, smart building and health care [1] [2]. WSN consists of a large number of sensor nodes which are supposed to constantly sense, supervise and control the surrounding environment. In sensor networks, wearable nodes are likely to be battery powered and thus rely on a limited energy supply to sense, process and communicate the data to the base station (BS) [3]. Consequently, it is of paramount importance to design energy aware protocols and algorithms, as replacing depleted batteries may be a costly and difficult operation.

Wireless data communication has been deemed the most critical energy demanding among other energy sources in sensor nodes [4]. For this reason, routing approaches have been recently considered as one of the crucial energy efficient techniques used in WSN to lower the communication energy burden. Cluster-based routing architectures have been widely used in WSN due to their energy efficiency and load balancing in the network [5]. Sensor nodes in cluster architecture are grouped into clusters in which a cluster head $(\mathrm{CH})$ is elected, and a group of source nodes are directly attached to the $\mathrm{CH}$. In recent works, various clustered routing protocols have been developed for the sake to prolong the longevity of the network [6][7]. However, most of these protocols are designed based on energy homogeneous nodes, which are supposed to have equal initial energy such as LEACH, PEGASIS and HEED [8][9][10]. 
Typically, sensor nodes are energy heterogeneous in many applications. For this reason, providing energy-centric heterogeneous routing protocols is highly required.

This paper provides a performance analysis of the most energy-aware heterogeneous clustered routing protocols based on Stable Election Protocol (SEP). To this end, we have thoroughly investigated the performances of routing protocols according to their energy efficiency, network lifetime, throughput and stability.

The rest of the paper is organized as follows. Section 2 describes the WSN system model and the radio energy model used. Section 3 discusses the main clustered routing protocols based on SEP algorithm and their performances. Section 4 shows the simulation results and discusses the performance of various SEP based protocols for heterogeneous WSN. Section 5 concludes the paper.

\section{System model}

We consider a heterogeneous sensor network composed by $\mathrm{N}$ sensor nodes that constantly monitor a phenomenon of interest and communicate data to a distant BS in a clustered fashion. Each sensor node in the network is assumed to be battery-powered with a limited energy supply. Sensor nodes access the channel in a Time Division Multiple Access (TDMA) method, in which time is partitioned into frames. Each frame corresponds to the time interval [tk; tk+1]. In each frame, nodes convey the sensed data to the attached $\mathrm{CH}$, and then to the $\mathrm{BS}$ which is assumed to be in the center of the network in order to reduce the communication distances. Moreover, nodes are supposed to be fix in the network without any mobility system.

\subsection{Radio energy model}

The wireless communication process is the most energy consuming in sensor networks compared to sensing and processing. The first order radio model is used in this work to evaluate the energy consumption of sensor nodes [8]. This energy model highlights the energy consumed in transmission, reception and circuitry. It depends mainly on the distance between transmitter and receiver, the packet size and the channel pathloss. Consequently, the energy required to transmit a $k$-bits information over a distance $d$ is given by

$$
E_{T x}(k, d)= \begin{cases}k \times E_{\text {elec }}+\varepsilon_{f s} \times d^{2}, & \text { if } d<d_{0} \\ k \times E_{\text {elec }}+\varepsilon_{m p} \times d^{4}, & \text { if } d \geq d_{0}\end{cases}
$$

Where $E_{\text {elec }}$ is the energy required to run the transmitter and receiver circuitry, $\varepsilon_{f s}$ and $\varepsilon_{m p}$ are the energies per bit used for the transmission amplifier in free space $\left(\approx d^{2}\right)$ and Two ray multipath $\left(\approx d^{4}\right)$, respectively, and $d$ is the distance between the transmitter and receiver. Note that when the inter- node distance $d<d_{0}$, the transmission energy is consumed according to the Free space model, while when $d \geq d_{0}$ the transmission energy follows the Two-ray ground model. The transition from Free space to Two-ray ground propagation is carried out according to a threshold distance $d_{0}$, which is expressed as function of both free space and two-ray amplifiers as follows: 


$$
d_{0}=\sqrt{\frac{\varepsilon_{f s}}{\varepsilon_{m p}}}
$$

Where $\varepsilon_{f s}$ and $\varepsilon_{m p}$ are respectively the power amplifier when using free space(fs) model and the multipath $(\mathrm{mp})$ model.

The energy consumption required for reception depends on the circuitry energy $E_{\text {elec }}$ and received packet size $k$, which is given by

$$
E_{R x}(k)=E_{\text {elec }} \times k
$$

\section{Clustered based routing}

Clustering has been recently considered a pivotal approach to save transmission energy in WSN [11][1]. Indeed, the sensor network is divided into several groups namely clusters. In each cluster, a cluster head is elected among nodes, and several nodes are attached to the elected CH. Accordingly, each cluster contains a $\mathrm{CH}$ and several member nodes, and the network is formed by a group of clusters. The member nodes sense the environment and forward the collected data to the $\mathrm{CH}$, which is supposed to process and transmit the aggregated data to the BS. The clustering process enables to balance the energy among all nodes in the network and extends the network lifetime. Figure 1 shows the typical mechanism of clustering that consists of clusters, cluster heads, cluster members and a distant BS.

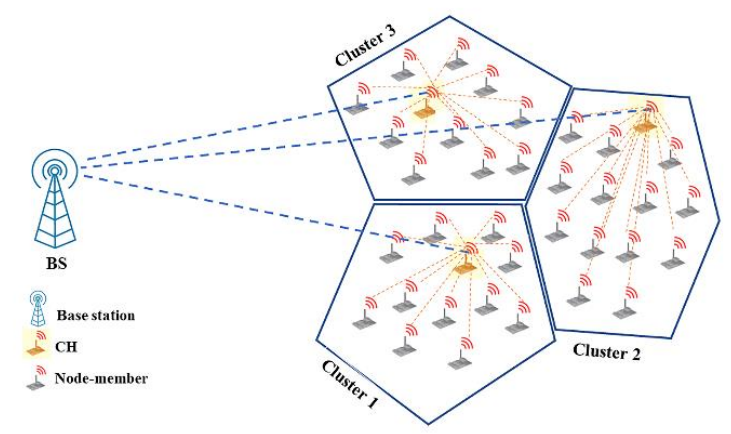

Fig. 1. Clustering technique in WSN

Among prior works, several approaches have been investigated to select efficiently the $\mathrm{CH}$. For instance, in LEACH protocol, a node is elected to be a CH based on a determined probability [11]. Other protocols are based on the energy level of nodes to decide 
the elected one. To this end, many clustered protocols have been developed according to various conditions of $\mathrm{CH}$ selection.

\subsection{Stable Election Protocol (SEP)}

SEP algorithm [12], improves the stable region of the hierarchy process by using the characteristic parameters of the heterogeneity, namely the fraction of the advanced nodes $m$ and the additional energy factor between the advanced and normal nodes $(\alpha)$. In order to extend the stable region, SEP tries to maintain the constraint of a well-balanced energy consumption. Intuitively, advanced nodes must become cluster leaders more often than normal nodes, which equate to a fairness constraint on energy consumption. The total energy of the new heterogenization is equal to:

$$
E_{\text {Total }}=n \times(1-m) \times E_{0}+n \times m \times E_{0} \times(1+\alpha)=n \times E_{0} \times(1+\alpha \times m)
$$

Where $n$ is total number of nodes, $m$ is the fraction of advanced nodes, $E_{0}$ is the supposed initial energy of nodes and $\alpha$ is the energy of advanced nodes. And the probabilities of normal and advanced nodes are respectively given by

$$
P_{n r m}=\frac{P_{o p t}}{1+\alpha \times m} \quad \text { (5) } \quad \text { and } \quad P_{a d v}=\frac{P_{o p t} \times(1+\alpha)}{1+\alpha \times m}
$$

Where $\boldsymbol{P}_{o p t}$ is the optimal probability of each node to become a $\mathrm{CH}$. Furthermore, those must satisfy the conditions below to be $\mathrm{CHs}$ in the network:

$$
\begin{aligned}
& T\left(s_{n r m}\right)= \begin{cases}\frac{P_{n r m}}{1-P_{n r m} \times\left(r \bmod \frac{1}{P_{n r m}}\right)}, & \text { if } s_{n r m} \in G^{\prime} \\
0 & , \quad \text { Otherwise }\end{cases} \\
& T\left(s_{a d v}\right)= \begin{cases}\frac{P_{a d v}}{1-P_{a d v} \times\left(r \bmod \frac{1}{P_{a d v}}\right)}, & \text { if } s_{a d v} \in G^{\prime \prime} \\
0 & \text { Otherwise }\end{cases}
\end{aligned}
$$

Where $G^{\prime}$ and $G^{\prime \prime}$ are the sets of nodes that have not been $\mathrm{CH}$ in last $\frac{1}{P_{n r m}}$ and $\frac{1}{P_{a d v}}$ rounds respectively for normal and advanced nodes, and $r$ is the associated round.

\subsection{Enhanced Stable Election Protocol (E-SEP)}

E-SEP [13], or enhanced type of the protocol SEP. Unlike SEP, this protocol deals with multi-level of sensor nodes in the network: normal, intermediate and advanced nodes. The total energy in the network is given by 


$$
\begin{aligned}
E_{\text {Total }} & =n \times(1-m-\lambda) \times E_{0}+n \times m \times E_{0} \times(1+\alpha)+n \times \lambda \times E_{0} \times(1+\mu) \\
& =n \times E_{0} \times(1+\alpha \times m+\lambda \times \mu)
\end{aligned}
$$

Where, $\alpha$ is the energy of advanced nodes, $\lambda$ is the proportion of intermediate nodes that have $\mu$ times more energy than normal nodes where $\mu=\frac{\alpha}{2}, n$ is total number of nodes, $m$ is the fraction of advanced nodes, $E_{0}$ is the supposed initial energy of nodes .

As in SEP, in E-SEP CHs are selected depending on probability of each type of node. Probabilities of becoming $\mathrm{CH}$ for normal, intermediate and advanced nodes, respectively are given by

$$
\begin{aligned}
P_{n r m} & =\frac{P_{o p t}}{(1+\alpha \times m+\lambda \times \mu)} \\
P_{\mathrm{int}} & =\frac{P_{o p t} \times(1+\alpha)}{(1+\mu)(1+\alpha \times m+\lambda \times \mu)} \\
P_{a d v} & =\frac{P_{o p t} \times(1+\alpha)}{(1+\lambda)(1+\alpha \times m+\lambda \times \mu)}
\end{aligned}
$$

\subsection{Zonal Stable Election Protocol (Z-SEP)}

Z-SEP shows two levels heterogeneity of nodes in the network as in SEP [14]. But the difference is that in Z-SEP normal nodes that are distributed near the BS use the direct transmission technique whereas advanced nodes use transmission via cluster heads. Generally, the network when using this technique is divided into three parties, zone 0 , zone 1 and zone 2 (See Figure 2). In Zone 0 located near the BS when normal nodes are distributed monitor their environment in transmit data directly to the BS. In both zone 1 and zone 2 , nodes use the clustering technique to send the aggregated data to the BS.

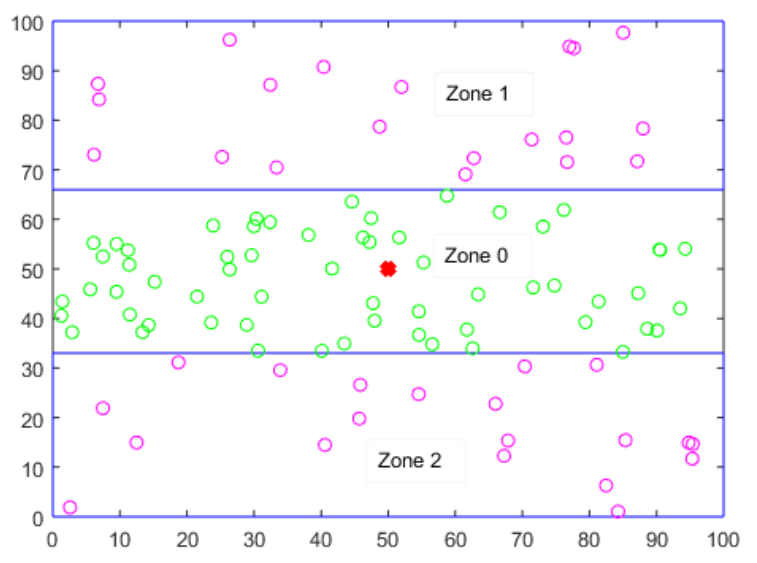

Fig. 2. The distribution of nodes in the network for the Z-SEP protocol 


\subsection{Threshold Stable Election Protocol (T-SEP)}

T-SEP [15] is also one of proactive protocols that uses three different levels of heterogeneity where nodes are classed into normal, intermediate and advanced. Moreover, the probabilities of being $\mathrm{CH}$ is the same as E-SEP. Whereas to ensure better selection of CHs, T-SEP takes into consideration a threshold for such type of nodes to become a cluster head when each node in the network generates randomly a number between 0 and 1 , if the generated value is less than threshold then this node becomes CHs. For each type of nodes, we got these different formulas for the calculation of threshold in function of their probabilities:

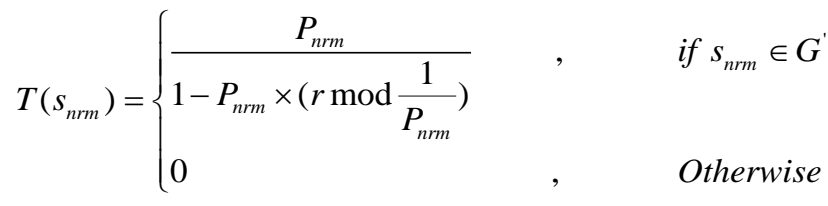

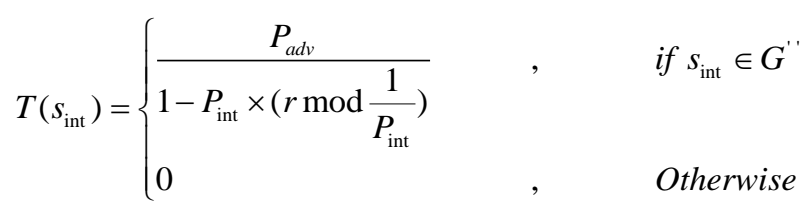

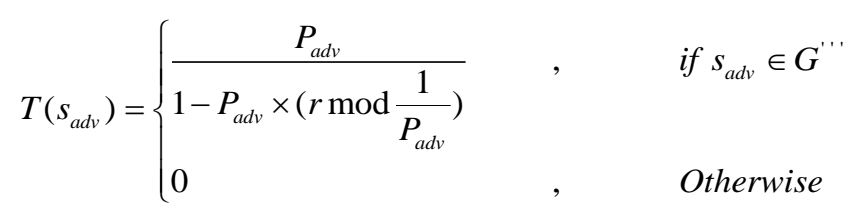

Where G', G', and G',' are the sets of nodes that have not been $\mathrm{CH}$ in last $\frac{1}{P_{n r m}}, \frac{1}{P_{\text {int }}}$ and $\frac{1}{P_{a d v}}$ rounds respectively for normal, intermediate and advanced nodes.

\section{Results and discussions}

In this section, we evaluate the performances of SEP based routing protocols according to various parameters such as energy efficiency, network lifetime, throughput and stability. A clustered WSN is implemented in Matlab simulator to evaluate the routing schemes. We have considered 100 heterogeneous sensor nodes randomly deployed in a square field of dimension $100 \mathrm{~m} \times 100 \mathrm{~m}$ and communicate with a BS which is located in the middle of the network. Nodes are assumed to have an initial energy level of $E_{o}=0.5 \mathrm{~J}$. The simulation parameters are listed in Table 1. For our simulations, to simplify, we did not take into consideration the energy loss caused by signal collision and interference in wireless channel. Furthermore, the energy value of normal nodes is obtained by adjusting the initial energy $E_{o}$ in function of the percentage of advanced and intermediate nodes in the network for each protocol. For the protocol Z-SEP, we choose $\mathrm{m}=0.4$ (40 \% of advanced nodes) with $\mathrm{a}=0.3$, and $60 \%$ of normal nodes. To get the energy value of normal nodes we did this calculation: $(50-E o(1+a)) / 60$. Although, for ESEP, where three levels of heterogeneity are required in the network, we choose $30 \%$ of nodes 
are advanced with $\mathrm{a}=0.3,20 \%$ of nodes are intermediate with $\mathrm{b}=\mathrm{a} / 2$ and $50 \%$ are normal, then we got the initial energy value of normal nodes by using this formula: $(50-\operatorname{Eo}(30 \times(1+a)+20 \times(1+b))) / 50$.

In addition, we assumed the following distributions of type of nodes: if the network contains two level of heterogeneity, we choose $60 \%$ of normal nodes and $40 \%$ of advanced ones, however, in three levels of heterogeneity we choose $50 \%$ of normal nodes, $30 \%$ of advanced nodes and $20 \%$ of super or intermediate nodes according to the case.

Table 1. Simulation parameters.

\begin{tabular}{ll}
\hline Parameter & Value \\
\hline Network's size & $100 \mathrm{~m} \mathrm{x} 100 \mathrm{~m}$ \\
Total number of nodes & 100 \\
Initial energy $E_{\mathrm{o}}$ & $0.5 \mathrm{~J}$ \\
Message size & $4000 \mathrm{bits}$ \\
$\mathrm{P}_{\mathrm{opt}}$ & 0.1 \\
Eelec $_{\mathrm{E}_{\mathrm{fs}}}$ & $50 \mathrm{~nJ} / \mathrm{bit}$ \\
$\mathrm{E}_{\mathrm{amp}}$ & $10 \mathrm{~nJ} / \mathrm{bit} / \mathrm{m}^{2}$ \\
Eda $_{\mathrm{da}}$ & $0.0013 \mathrm{pJ} / \mathrm{bit} / \mathrm{m}^{2}$ \\
Soft Threshold & $5 \mathrm{~nJ} / \mathrm{bit} / \mathrm{signal}$ \\
Hard Threshold & 100 \\
\hline
\end{tabular}

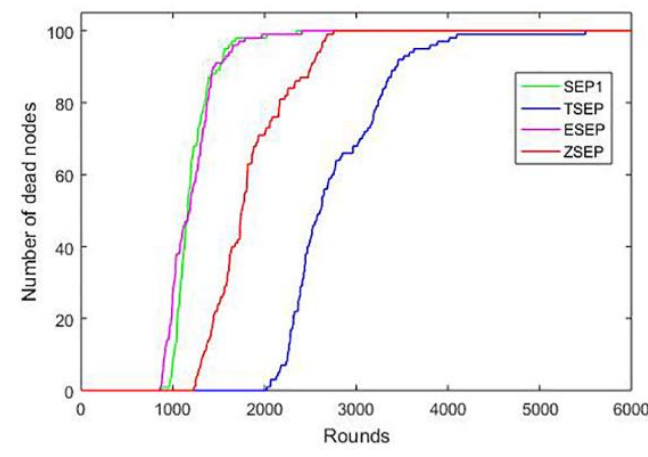

Fig. 3. Number of dead nodes versus rounds for SEP, T-SEP, E-SEP and Z-SEP

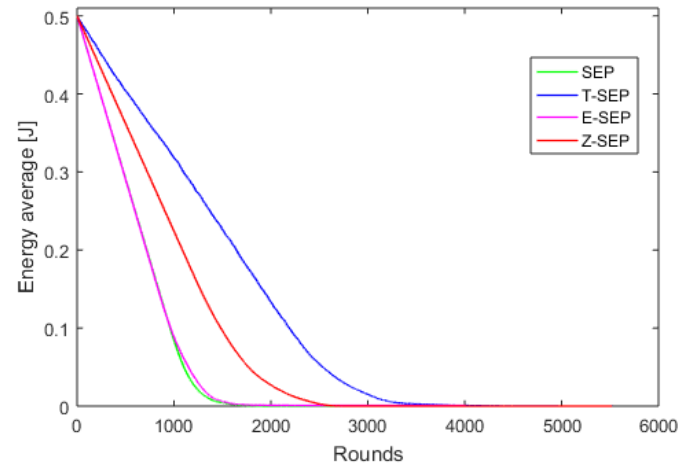

Fig. 4. Residual energy average of the network versus rounds for SEP, T-SEP, ESEP and Z-SEP 


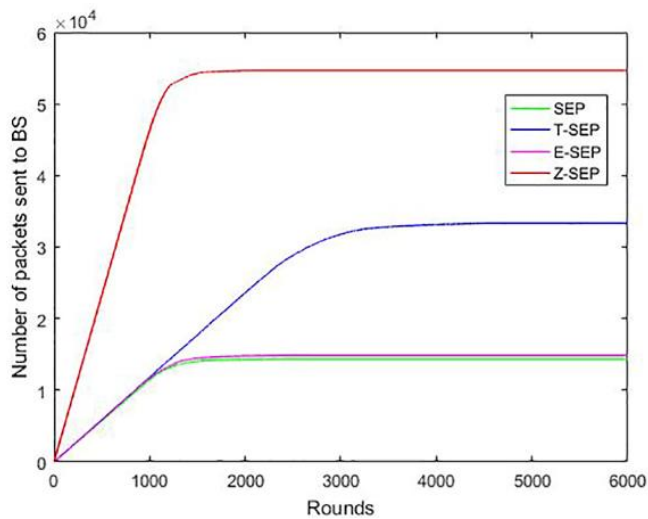

Fig. 5. Number of packets received by the BS using SEP, T-SEP, E-SEP and Z-SEP

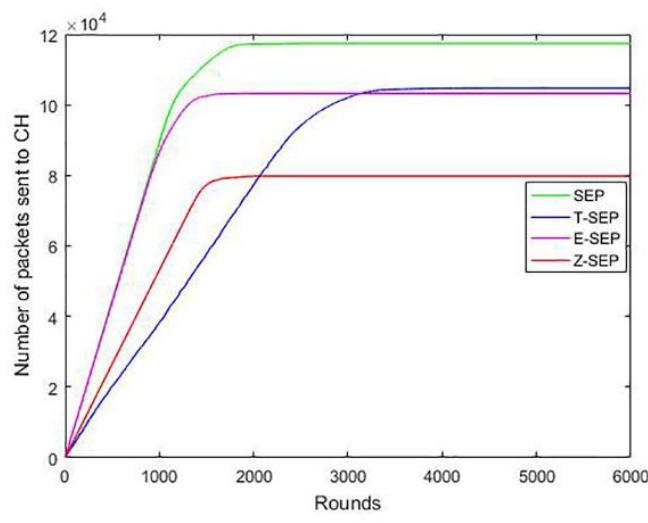

Fig. 6. Number of packets received by $\mathrm{CH}$ using SEP, T-SEP, E-SEP and Z-SEP

The average energy and number of dead nodes versus transmission rounds are shown in Figures 3 and 4, respectively. These Figures compare the performance of the studied routing algorithms i.e., SEP, E-SEP, Z-SEP and T-SEP. Table 2 summarizes the rounds when 1\%, 50\% and $100 \%$ of nodes are dead as well as the stability period of each protocol. The obtained results show that T-SEP performs better than the other protocols in terms of energy and network lifetime. When using T-SEP protocol, the percentages of $1 \%, 50 \%$ and $100 \%$ of dead nodes occur at 2026, 2604 and 5504 rounds, respectively. This corresponds to lifetime increase of 56 $\%, 55 \%$ and $57 \%$, respectively, when compared to SEP protocol. Besides, T-SEP protocol outclasses SEP, Z-SEP and E-SEP in terms of stability as it lasts 2026 rounds before nodes start dying compared to 1228,879 and 859 rounds when using Z-SEP, SEP and E-SEP, respectively. Figures 5 and $\mathbf{6}$ show the number of packets transmitted successfully to the $\mathrm{CH}$ and BS, respectively. This is to assess the throughput performance of the studied SEP based protocols in heterogeneous WSN. Table 3 provides the maximum number of packets sent to corresponding $\mathrm{CH}$ and $\mathrm{BS}$ for each protocol. The results depict that SEP provides the best throughput at intra clusters (i.e. 117408 packets), flowed by T-SEP (i.e. 104767 packets), E-SEP (i.e. 103275 packets) and then Z-SEP (i.e. 5452 packets). For the throughput to the BS, Z-SEP is proved to have the best performance (i.e. 54673 packets) compared to T-SEP (i.e. 33328), E-SEP (i.e. 14838) and then SEP (i.e. 14360).

As we have noticed the SEP protocol has a large number of packets transmitted to the cluster heads, this is explained by the fact that this one uses a large number of clusters, which also means a large number of $\mathrm{CH}$ nodes and finally a reduction in the number of packets transmitted by the member nodes to their $\mathrm{CHs}$ in the associated cluster. More precisely, if there are many clusters in the network, it means that there are many cluster heads, which also means fewer member nodes (no $\mathrm{CH}$ nodes), therefore fewer packets transmitted to $\mathrm{CH}$ and more packets transmitted to the BS. Conversely, if there are few clusters, then few cluster heads, which means many non-cluster head nodes and consequently many packets are transmitted to the $\mathrm{CHs}$ and fewer packets are transmitted to the BS. However, the best performances of throughput to the BS provided by Z-SEP is explained by the fact that $60 \%$ of nodes deployed in zone 0 (Figure 2) transmit their aggregated data directly to the BS. Indeed, the other $40 \%$ of nodes are distributed equally into two groups zone 1 and zone 2 exactly $20 \%$ in each one. That 
means a smaller number of CHs in those two zones, consequently less packets transmitted in a cluster by member nodes to their $\mathrm{CH}$ which explains likewise the poor performance of Z-SEP represented in red curve in Figure 6. Moreover, when the network losses all its sensor nodes, this means immediately no packets transmissions are occurred whether to their $\mathrm{CH}$ or to $\mathrm{BS}$, the fact that explains the curves stabilization in Figures 5 and $\mathbf{6}$.

Generally, the nodes energy consumption is inversely proportional to the number of rounds ran down. Moreover, in first rounds, all nodes in the network are able to communicate but just after certain rounds, some ones become unable to carry out a radio communication operation due to insufficient energy residual in their batteries.

Then, round after round the network continues to lose node after node till the last one. This operation of node loss in the network after the course of the cycles affects also the number of packets transmitted whatsoever towards the $\mathrm{CHs}$ or to the BS. More precisely, in our simulation the number of packets transmitted to $\mathrm{CH}$ by T-SEP in the first round is 93 packets although only 23 packets are transmitted in round number 3000 which means a drop of $75.26 \%$ of performance carried out in the first round after 3000 rounds. On the other hand, 7 packets are transmitted to the BS in the first round as well just 5 packets are transmitted in the round number 3000 which also signifies a decrease of $28.57 \%$ of performance showed in the first round.

Table 2. Performance comparison of SEP based routing protocols

\begin{tabular}{lllll}
\hline Protocol & SEP & Z-SEP & E-SEP & T-SEP \\
\hline First node dead & 879 & 1228 & 859 & 2026 \\
$50 \%$ of dead nodes & 1162 & 1747 & 1178 & 2604 \\
$100 \%$ of dead nodes/Network lifetime & 2350 & 2726 & 2411 & 5504 \\
Stability period & 879 & 1228 & 859 & 2026 \\
\hline
\end{tabular}

Table 3. Number of transmitted packets to cluster heads and to the BS

\begin{tabular}{lllll}
\hline Protocol & SEP & Z-SEP & E-SEP & T-SEP \\
\hline Max Nu. of packet sent to CH & 117408 & 5452 & 103275 & 104767 \\
Max Nu. of packet sent to BS & 14360 & 54673 & 14838 & 33328 \\
\hline
\end{tabular}

\section{Conclusion}

In this work, an extensive analysis of routing protocols based on SEP in heterogeneous WSN is presented. This paper has mainly focused on the imminent SEP based routing protocols dedicated for clustered architecture, namely, SEP, E-SEP, T-SEP and Z-SEP. We have provided a performance comparison of the studied routing protocols based on their energy efficiency, network lifetime, throughput and stability. The simulation results have shown that the T-SEP protocol outperforms SEP, Z-SEP and E-SEP in terms of energy efficiency, network lifetime and stability. However, SEP and T-SEP have proved to be efficient than Z-SEP and E-SEP in terms of throughput at $\mathrm{CH}$ nodes and BS, respectively. Consequently, T-SEP can be a suitable choice for energy constrained heterogeneous WSN in order to save energy and prolong the network lifespan. 


\section{References}

[1] A. Berl et al., "Energy-efficient cloud computing," Comput. J., vol. 53, no. 7, pp. 1045$1051,2010$.

[2] I. F. Akyildiz, W. Su, Y. Sankarasubramaniam, and E. Cayirci, "A survey on sensor networks," IEEE Commun. Mag., vol. 40, no. 8, pp. 102-114, 2002.

[3] E. Fasolo, M. Rossi, J. Widmer, and M. Zorzi, "In-network aggregation techniques for wireless sensor networks: a survey," IEEE Wirel. Commun., vol. 14, no. 2, 2007.

[4] N. A. Pantazis and D. D. Vergados, "A survey on power control issues in wireless sensor networks," IEEE Commun. Surv. Tutorials, vol. 9, no. 4, pp. 86-107, 2007.

[5] S. Bandyopadhyay and E. J. Coyle, "An energy efficient hierarchical clustering algorithm for wireless sensor networks," in INFOCOM 2003. Twenty-Second Annual Joint Conference of the IEEE Computer and Communications. IEEE Societies, 2003, vol. 3, pp. 1713-1723.

[6] S. Tanwar, N. Kumar, and J. J. P. C. Rodrigues, "A systematic review on heterogeneous routing protocols for wireless sensor network," J. Netw. Comput. Appl., vol. 53, pp. 39-56, 2015.

[7] A. Mahboub, M. Arioua, I. Ez-Zazi, and A. El Oualkadi, "Multi-zonal approach clustering based on stable election protocol in heterogeneous wireless sensor networks," in Information Science and Technology (CiSt), 2016 4th IEEE International Colloquium on, 2016, pp. 912 917.

[8] M. Lauridsen, B. Vejlgaard, I. Z. Kovacs, H. Nguyen, and P. Mogensen, "Interference measurements in the European $868 \mathrm{MHz}$ ISM band with focus on LoRa and SigFox," in Wireless Communications and Networking Conference (WCNC), 2017 IEEE, 2017, pp. 16.

[9] S. Lindsey and C. S. Raghavendra, "PEGASIS: Power-efficient gathering in sensor information systems," in Aerospace conference proceedings, 2002. IEEE, 2002, vol. 3, p. 3.

[10] O. Younis and S. Fahmy, "HEED: a hybrid, energy-efficient, distributed clustering approach for ad hoc sensor networks," IEEE Trans. Mob. Comput., vol. 3, no. 4, pp. 366379, 2004.

[11] W. R. Heinzelman, A. Chandrakasan, and H. Balakrishnan, "Energy-efficient communication protocol for wireless microsensor networks," in System sciences, 2000. Proceedings of the 33rd annual Hawaii international conference on, 2000, p. 10-pp.

[12] G. Smaragdakis, I. Matta, and A. Bestavros, "SEP: A stable election protocol for clustered heterogeneous wireless sensor networks," Boston University Computer Science Department, 2004.

[13] S. Faisal, N. Javaid, A. Javaid, M. A. Khan, S. H. Bouk, and Z. A. Khan, "Z-SEP: Zonalstable election protocol for wireless sensor networks," arXiv Prepr. arXiv1303.5364, 2013.

[14] S. Faisal, N. Javaid, A. Javaid, M. A. Khan, S. H. Bouk, and Z. A. Khan, "Z-SEP: Zonalstable election protocol for wireless sensor networks," arXiv Prepr. arXiv1303.5364, 2013.

[15] A. Kashaf, N. Javaid, Z. A. Khan, and I. A. Khan, "TSEP: Threshold-sensitive stable election protocol for WSNs," in Frontiers of Information Technology (FIT), 2012 10th International Conference on, 2012, pp. 164-168. 